\title{
RETRACTED ARTICLE: Radius of convergence of $p$-adic connections and the Berkovich ramification locus
}

\section{Francesco Baldassarri}

Received: 2 March 2012 / Revised: 10 May 2012 / Published online: 13 November 2012 (C) Springer-Verlag Berlin Heidelberg 2012

This paper has been retracted by the author because of an error. Proposition 3.13 is false and leads to erroneous statements in Theorem 0.8 and its corollary 0.11 and the last sentence of Theorem 0.1. 